\section{THE 1992 IMS SEPTEMBER MEETING}

\author{
Michael Brennan and Brendan McCann
}

The 1992 September meeting took place on Thursday 3rd and Friday 4 th September at Waterford Regional Technical College, and was organized by the mathematics staff at WRTC. There were over 40 participants at the meeting, and it was very encouraging to note that many of the lectures were attended by computer science, engineering and physics staff at WRTC.

After the opening address by the principal of WRTC, Mr Ray Griffin, the first speaker was Professor Darrel Ince (Department of Computing, Open University). In his talk, Discrete mathematics and the formal development of programs, he outlined some of the advances and retreats of formal (that is, mathematical) methods of program specification and verification. The second talk of the opening session was given by Dr John McDermott (UCG). It was entitled Colouring problems, and in it he described some of the problems involved in determining the chromatic number (that is, the minimum number of colours needed to distinguish the vertices) of a directed graph.

After lunch on the first day, Professor David Armitage (QUB) spoke on Harmonic functions: background and recent results. In his talk he reviewed the development of the theory of harmonic functions, and included an account of some of the new results of the past few years. Then Mr Eamonn de Leastar (WRTC) presented a talk on Numerical data types in $C$ and $C^{++}$, in which he discussed the differences between the two languages, using as an example a simple program specification.

There followed a coffee break, after which Professor Gilbert Strang (Massachusetts Institute of Technology) discussed the case of Wavelet transforms vs. Fourier transforms. After presenting examples, an application to high resolution television, and a review of the mathematical theory, he concluded that the Fast Fourier Transform will not, in most cases, be superseded by the Fast Wavelet Transform. The final talk of the first day, Numerical solution of convection-diffusion problems, was given by Dr Martin Stynes (UCC). He talked about some of the very difficult numerical problems encountered when trying to approximate the solution to the second order differential equations which model physical situations with "dominant" convection and secondary diffusion.

The second day of the meeting began with Higher order symmetry of graphs, a talk given by Professor Ronald Brown (University of North Wales, Bangor). He described a category theoretical approach to the idea of symmetry, involving the category of digraphs, appropriate morphisms, and the general notion of a topos in place of a set. Then Professor Joaquín Gutiérrez (Università Politecnica di Madrid) talked on Polynomials and series in Banach spaces, and dealt with those series in Banach spaces whose convergence is preserved under certain polynomial mappings. After coffee, Professor John Lewis (DIAS) spoke on Thermodynamic aspects of probability theory. He discussed Boltzmann's equation: $S=k \log W$ (where $S$ is the entropy of an equilibrium state, and $W$ is the number of microscopic states corresponding to that equilibrium state), and the corresponding probability theory namely Large Deviation Theory.

The afternoon session began with Professor Strang's second talk New ideas on teaching calculus, linear algebra and applied mathematics, in which he gave a flavour of his own teaching style by going through a number of easy-to-follow examples. Then Dr Patrick Fitzpatrick (UCC) talked about $A$ theoretical basis for Padé approximation, where the problem is to approximate a polynomial of given degree by the quotient of 2 polynomials of lesser degree. He discussed applications to coding theory, and showed how Gröbner bases help to resolve some of the problems.

After coffee Mr Christopher Boyd (UCD) gave a talk on Preduals and linearization of holomorphic mappings, which dealt with dual-nuclear spaces of holomorphic functions. The meeting then 
closed with an address by the President of the IMS, Dr Richard Timoney.

The organizers would like to thank all the speakers and participants at the meeting, especially those who chaired the sessions. In addition they would like to thank Waterford Regional Technical College and the City of Waterford Vocational Education Committee for their generous funding, and the Waterford branch of the Bank of Ireland who provided a contribution towards the running costs of the meeting.

Michael Brennan and Brendan McCann

Regional Technical College

Waterford

\section{THE CONTINUITY \\ OF THE SEMI-FREDHOLM INDEX}

Mícheál Ó Searcóid

\section{Introduction}

It is well-known and easy to prove that the index function is continuous on the set of Fredholm operators on a Banach space. It is also true that the index function is continuous on the larger set of semi-Fredholm operators. The proof presents no difficulty in the case where the semi-Fredholm operators are simply those operators which are left or right invertible modulo the compact operators, as happens in the case where the Banach space is a Hilbert space. The usual proofs in the more general context [4, p.60]; $[1$, pp.62-63] use the notion of gap between between subspaces and require more preliminary work than one might have suspected necessary. In this note we show how to avoid such unsatisfactory excursions by giving a natural operator-theoretic proof of this basic result. The nature of the proof makes it convenient to consider the slightly more general case in which the operators act between two possibly different spaces.

\section{Preliminaries}

If $X$ and $Y$ are Banach spaces, then $\mathcal{B}(X, Y)$ will denote the set of bounded linear operators from $X$ to $Y$, and $\mathcal{F}(X, Y)$ will denote the set of finite rank operators in $\mathcal{B}(X, Y)$. When $X=Y$ we shall write $\mathcal{B}(X)$ instead of $\mathcal{B}(X, Y)$ and $\mathcal{F}(X)$ instead of $\mathcal{F}(X, Y)$; all similar notation will be abbreviated in the same way. Identity operators on spaces will be denoted by $I$; the space in question will always be obvious from the context. For each $T \in \mathcal{B}(X, Y)$, the nullity $\operatorname{nul}(T)$, and the defect $\operatorname{def}(T)$ of $T$ are defined to be 